\title{
Laser-triggered release of encapsulated molecules from polylactic-co-glycolic acid microcapsules
}

Kazumasa Ariyasu

Atsuhiro Ishii

Taiga Umemoto

Mitsuhiro Terakawa 


\title{
Laser-triggered release of encapsulated molecules from polylactic-co-glycolic acid microcapsules
}

\author{
Kazumasa Ariyasu, ${ }^{a, b}$ Atsuhiro Ishii, ${ }^{b}$ Taiga Umemoto, ${ }^{b}$ and Mitsuhiro Terakawa ${ }^{a, b, *}$ \\ ${ }^{a}$ Keio University, Department of Electronics and Electrical Engineering, 3-14-1 Hiyoshi, Kohoku-ku, Yokohama, Kanagawa 223-8522, Japan \\ ${ }^{b}$ Keio University, School of Integrated Design Engineering, 3-14-1 Hiyoshi, Kohoku-ku, Yokohama, Kanagawa 223-8522, Japan
}

\begin{abstract}
The controlled release of encapsulated molecules from a microcapsule is a promising method of targeted drug delivery. Laser-triggered methods for the release of encapsulated molecules have the advantage of spatial and temporal controllability. In this study, we demonstrated the release of encapsulated molecules from biodegradable polymer-based microcapsules using near-infrared femtosecond laser pulses. The polylactic-coglycolic acid microcapsules encapsulating fluorescein isothiocyanate-dextran molecules were fabricated using a dual-coaxial nozzle system. Irradiation of femtosecond laser pulses enhanced the release of the molecules from the microcapsules, which was accompanied by a decrease in the residual ratio of the microcapsules. The laserinduced modification of the surface of the shell of the microcapsules indicated the potential for sustained release as well as burst release. (๑) 2016 Society of Photo-Optical Instrumentation Engineers (SPIE) [DOI: 10.1117/1.JBO.21.8.085003]
\end{abstract}

Keywords: biodegradable polymer; microcapsule; femtosecond laser; drug release; drug delivery.

Paper 160428R received Jun. 21, 2016; accepted for publication Aug. 9, 2016; published online Aug. $23,2016$.

\section{Introduction}

The controlled release of encapsulated molecules from a microcapsule has been widely investigated as a drug delivery system (DDS) ${ }^{1-3}$ The encapsulation prevents the decomposition of the drug molecules in blood, resulting in the successful delivery of molecules such as proteins and nucleic acids to the targeted site. For example, microcapsules with a diameter of approximately 1 to $5 \mu \mathrm{m}$ were investigated for use as carriers for the targeting of antigen-presenting cells, whereas microcapsules with a diameter larger than $10 \mu \mathrm{m}$ were used in the chemoembolization of carcinoma. ${ }^{4-6}$ Biodegradable polymers are promising materials for such microcapsules because of their low toxicity and degradable properties in the human body. ${ }^{7-10}$ Polylactic-co-glycolic acid (PLGA) is one of the most attractive biodegradable polymers that the FDA has approved for clinical use. The release property of the PLGA microcapsules depends on the molecular weight and crystallinity of the PLGA, the ratio of polylactic acid (PLA) to polylactic glycolic acid (PGA), and the encapsulated molecules and the concentration of the encapsulated molecules. ${ }^{11-13}$ The application of PLGA microcapsules in a DDS is not limited to use as a conventional drug carrier; it may also be embedded in a drug-releasing scaffold, which has recently been reported. ${ }^{14}$

The pharmacological effect will be maximized if the drug release from a microcapsule can be controlled by an external stimulus at a targeted site at a stipulated time, which will also help minimize side effects. The use of ultrasound, ${ }^{15}$ change in $\mathrm{pH},{ }^{16}$ and electric field ${ }^{17}$ as stimuli have been reported in previous studies. Among others, laser-mediated methods are attractive in regard to spatial and temporal controllability. Near-infrared (NIR) wavelengths can be used as a trigger for drug release from deeply located microcapsules because of the deep penetration depth of NIR in tissues owing to its low absorption. Since most of the polymer microcapsules demonstrate low NIR

*Address all correspondence to: Mitsuhiro Terakawa, E-mail: terakawa@elec. keio.ac.jp absorption, gold nanorods were used as absorbers. ${ }^{18}$ In our previous study, we demonstrated the perforation of hollow polystyrene microcapsules using an NIR femtosecond laser without any doping with absorbing metals or dyes that may cause cell toxicity. ${ }^{19}$ The simulation results indicated that the enhancement of optical intensity on the shell of a microcapsule contributed to the nanoperforation of the shell. However, the previous results were only limited to the demonstration of rupture on the commercially available polystyrene microcapsules.

In this study, we demonstrated the release of encapsulated molecules from PLGA microcapsules using an NIR femtosecond laser as a method of drug release. The microcapsules were fabricated using a dual-coaxial nozzle system. The residual ratio of the microcapsules and fluorescence molecules released from the microcapsules was evaluated. The surface of the shell was observed using scanning electron microscopy (SEM) in order to discuss the mechanism and the potential of the release.

\section{Materials and Methods}

\subsection{Fabrication of PLGA Microcapsules}

Poly (L-lactide-co-glycolide) (PLGA) (lactic acid:glycolic acid $=50: 50$, i.v. $=0.26 \mathrm{dl} / \mathrm{g}, \mathrm{MW}=28,000$, as provided by the manufacturer) was obtained from BMG Inc. (Kyoto, Japan). Polyvinyl alcohol (PVA) and fluorescein isothiocyanate-dextran (FITC-dextran) (2 MDa) were purchased from Wako Pure Chemical Industries, Ltd. and Sigma-Aldrich Co. LLC., respectively.

The PLGA microcapsules were fabricated using the method reported by Berkland et al. ${ }^{8,9} 1 \%(\mathrm{w} / \mathrm{v})$ PLGA dissolved in dichloromethane (DCM) and $0.1 \%$ (w/v) FITC-dextran dissolved in distilled water were prepared. The PLGA suspension and FITC-dextran solution were pumped into the outer and inner nozzles of a dual-coaxial nozzle at a flow rate of 166.67 and

$1083-3668 / 2016 / \$ 25.00$ @ 2016 SPIE 
$100.00 \mathrm{ml} / \mathrm{h}$, respectively. The stream flowing out of the nozzle was split into uniform droplets by the vibrations from a clamped ultrasonic transducer $(0.028 Z 30$ N, Japan Probe Co., Ltd., Yokohama, Japan) that was controlled by a frequency generator (Agilent 33220A, Agilent Technologies, Santa Clara, California). The droplets flowed into a beaker containing $100 \mathrm{ml}$ of $0.05 \%$ PVA. To evaporate the DCM, the suspension containing the droplets was stirred overnight with a magnetic stirrer. The fabricated PLGA microcapsules containing FITCdextran were observed using SEM and fluorescence microscopy (Eclipse Ti, Nikon, Tokyo, Japan). The microcapsules were placed in distilled water and kept in a $\mathrm{CO}_{2}$ incubator until the SEM observation was conducted. The average diameter of the PLGA microcapsules was determined by measuring 50 randomly selected microcapsules.

\subsection{Laser Irradiation of Microcapsules}

A Ti:sapphire chirped-pulse amplification laser system (Libra, Coherent Inc., Santa Clara, California), which generates linearly polarized 80-fs laser pulses at a central wavelength of $800 \mathrm{~nm}$ and a repetition rate of $1 \mathrm{kHz}$, was used in the experiments. The fabricated PLGA microcapsules, which were suspended in $3.5 \mu \mathrm{l}$ of distilled water, were dripped into a glass-bottomed dish (27 $\mathrm{mm}$ in diameter) and sandwiched with a cover glass sheet $(0.15 \mathrm{~mm}$ in thickness) to make the surface of the suspension perpendicular to the laser incidence. The distance between the cover glass sheet and the bottom of the dish, i.e., height of the suspension, was $1.25 \mathrm{~mm}$. The microcapsules were irradiated with multiple laser pulses from the top of the dish. The laser beam was loosely focused with a plano-convex lens $(f=200 \mathrm{~mm})$ to a spot diameter of $300 \mu \mathrm{m}$ and scanned to irradiate the entire suspension.

\subsection{Residual Ratio of Microcapsules}

After laser irradiation, the distilled water was evaporated and the number of microcapsules in a dish was counted carefully through microscopic observation. The residual ratio of the microcapsules, $N_{2} / N_{1}$, was determined, where $N_{1}$ and $N_{2}$ are the numbers of microcapsules before and after laser irradiation, respectively. The average number of microcapsules before laser irradiation was 309 per dish.

\subsection{Release of Encapsulated Molecules from PLGA Microcapsules}

Six hundred microliters of distilled water containing PLGA microcapsules ( 88.3 capsules $/ \mu \mathrm{l}$ ) was placed in a cover glass chamber slide, segmented to $10 \mathrm{~mm} \times 20 \mathrm{~mm}$ spaces. Femtosecond laser pulses were irradiated to the top side of the cover glass chamber side, in a configuration identical to that described in Sec. 2.2; however, the diameter of the laser pulse was $600 \mu \mathrm{m}$. The laser beam scanned the cover glass chamber side at a speed of $2 \mathrm{~mm} / \mathrm{s}$. The suspension was centrifuged for $10 \mathrm{~min}$ at $10,000 \mathrm{rpm}$ after laser irradiation, so that the supernatant solution could be collected with the released fluorescent molecules from the microcapsules. The fluorescence measurement was performed for the wavelength at $518 \mathrm{~nm}$, with the excitation wavelength at $452 \mathrm{~nm}$. The suspension that did not undergo laser irradiation was also evaluated with the same protocol as the control condition (laser fluence of $0 \mathrm{~mJ} / \mathrm{cm}^{2}$ ). The effect of fluorescence photobleaching caused by laser irradiation was also evaluated. Six hundred microliters of the $0.10 \%$ (w/v) FITC-dextran solution was irradiated by the laser pulses in the chamber slide.

\subsection{Simulation of Optical Intensity Around PLGA Microcapsules}

The numerical analysis of the optical intensity was performed by the three-dimensional (3-D) finite-difference time-domain (FDTD) method. The simulation model consisted of a PLGA microsphere $(n=1.59)$ which had outer and inner diameters of 22.5 and $11.1 \mu \mathrm{m}$, respectively. The diameters were determined based on the average diameters of the fabricated microcapsules in the experiments. The surrounding and the inner core of the microcapsule were set as filled with water $(n=1.33)$. The linearly polarized plane wave at a wavelength of $800 \mathrm{~nm}$ was incident from the top of the microcapsule.

\subsection{Statistical Analysis}

The experiments on the release of encapsulated molecules from the PLGA microcapsules were performed for five samples in each condition $(n=5)$. Values of $p<0.01$ were determined as statistically different based on the Student's $t$-test (unpaired).

\section{Results}

Figure 1 shows the microscopic images of the fabricated PLGA microcapsules. The SEM image of the fabricated microcapsules is shown as Fig. 1(a), which shows that the spherical microcapsules were fabricated. Several dimples smaller than $1 \mu \mathrm{m}$ were observed on the magnified image of the shell surface [Fig. 1(b)]. Figure 1(c) shows the phase contrast and fluorescent images of the microcapsules. Figures 1(a)-1(c) were obtained with the ultrasound frequency at $150 \mathrm{kHz}$ for the vibration of the nozzle. The fluorescence of FITC-dextran was observed inside the shell of almost all the microcapsules, as shown in Fig. 1(c), indicating that the fluorescence molecules were encapsulated inside microcapsules. Figure 2(a) shows the dependence of the diameter of the microcapsules on the ultrasound frequency. There is a sharp fall in the experimental values of the microcapsule diameter when the ultrasound frequency is lower than $10 \mathrm{kHz}$, which is then followed by a moderate decrease in microcapsule diameter up to $150 \mathrm{kHz}$. The theoretical diameter $r_{t}$ is also shown in the figure, which was calculated by assuming that spherical droplets were formed:

$r_{t}=\left[\frac{3\left(v_{f}+v_{p}\right)}{4 \pi f}\right]^{\frac{1}{3}}$,

where $v_{f}$ and $v_{p}$ are the flow volumes of the FITC-dextran solution and PLGA suspension, respectively, and $f$ indicates the ultrasound frequency. From the results, we used an ultrasound frequency of $150 \mathrm{kHz}$, which realized comparably smaller diameters, for the following experiments. Figure 2(b) shows the histogram of the microcapsule diameter distribution. The average diameter of the microcapsules was $22.5 \pm 0.8 \mu \mathrm{m}$ at $150 \mathrm{kHz}$.

Figure 3 shows the residual ratios of the microcapsules after femtosecond laser irradiation. The residual ratio shows a decrease with increasing laser fluence under the scanning velocity, which is fixed at $1 \mathrm{~mm} / \mathrm{s}$ [Fig. 3(a)]. The effect of the number of laser pulses on the residual ratio is also evaluated by changing the scanning velocity from 0.3 to $3.0 \mathrm{~mm}$, corresponding to the 

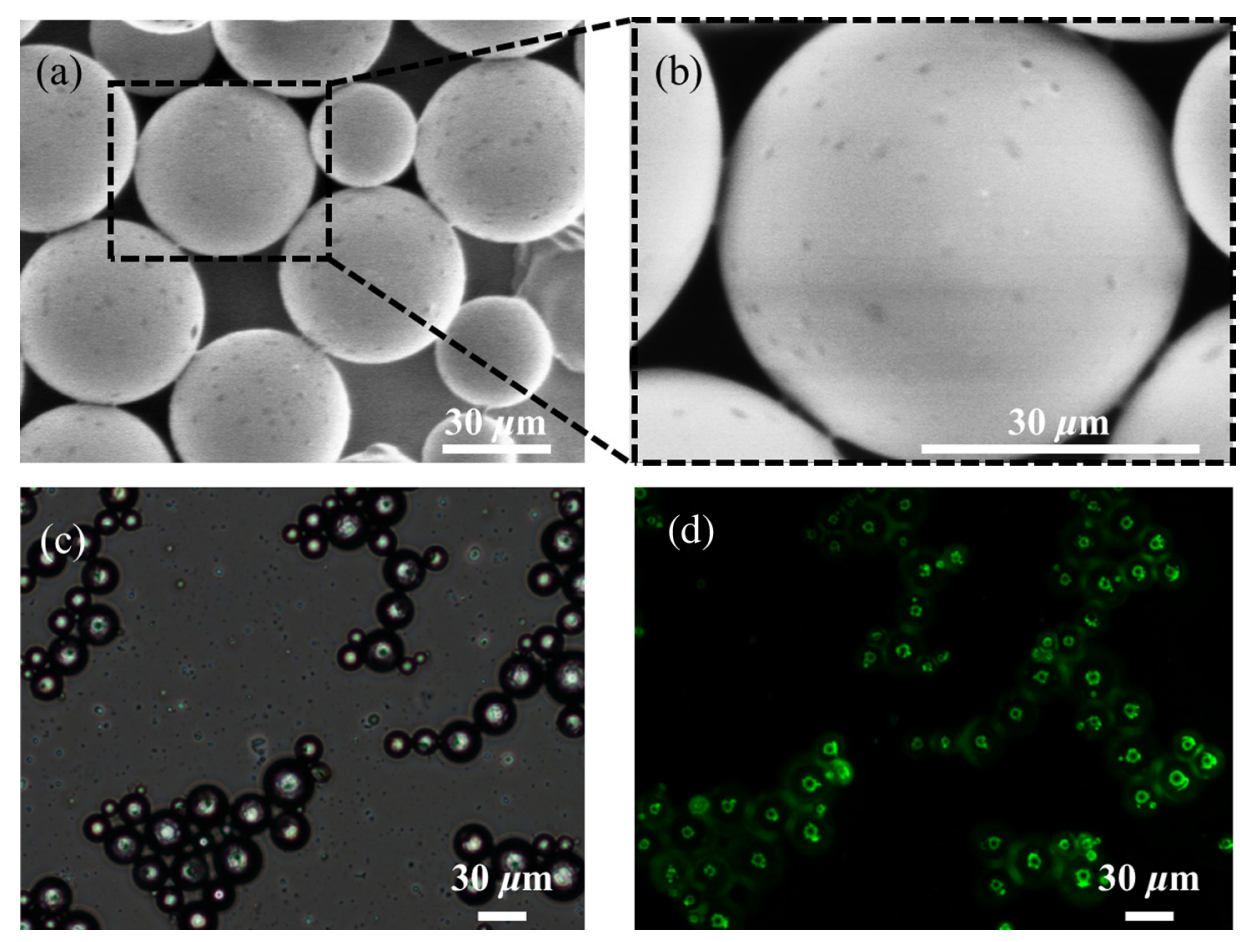

Fig. 1 Fabricated PLGA microcapsules encapsulating FITC-dextran. (a) SEM image of the microcapsules. (b) Enlarged image of the dashed square in (a). (c) Phase contrast and (d) fluorescence images of the fabricated microcapsules. The microcapsules were fabricated with the ultrasound frequency of $150 \mathrm{kHz}$.

(a)

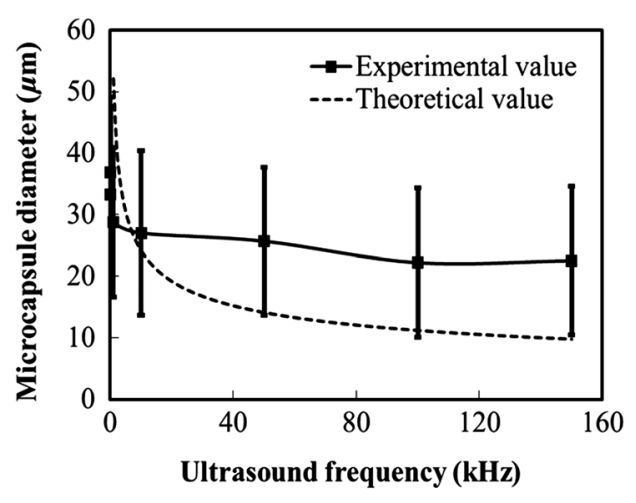

(b) 1

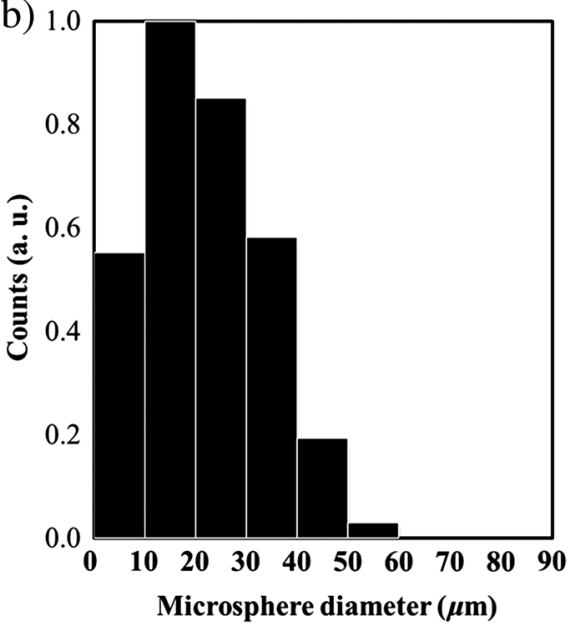

Fig. 2 (a) Dependence of the diameter of the fabricated microcapsules on the ultrasound frequency. The theoretical diameter is also shown as a dashed line. Error bars indicate standard deviation. (b) Histogram of the microcapsule diameter distribution at the ultrasound frequency of $150 \mathrm{kHz}$.

pulse overlaps from 1000 to 100 pulses. The result shows that the scanning velocity is negligible under different laser fluences of 100,200 , and $300 \mathrm{~mJ} / \mathrm{cm}^{2}$, as shown in Fig. 3(b). The residual ratios were evaluated by optical microscopic observation, therefore, only the spherical microcapsules were counted for the figures. We have observed fragments of PLGA microcapsules after laser irradiation at following SEM observations.

The result of the release of the fluorescence molecules from the microcapsules is shown in Fig. 4. The fluorescence intensity was normalized to the value of the control samples (without laser irradiation, $0 \mathrm{~mJ} / \mathrm{cm}^{2}$ ). The increase in laser fluence enhanced the release of the fluorescence molecules. Fluorescence intensities at the laser fluences of 200 and $300 \mathrm{~mJ} / \mathrm{cm}^{2}$ are statistically significant to the fluorescence intensities of the control samples.

The surface of the microcapsule was observed by SEM on the second and sixth days after the fabrication. Figures 5(a) and 5(b) show the microcapsules irradiated by femtosecond laser pulses. The microcapsules were irradiated 1 day after the fabrication; Figs. 5(a) and 5(b) show the microcapsules on the first and fifth days after laser irradiation, respectively. 
(a)

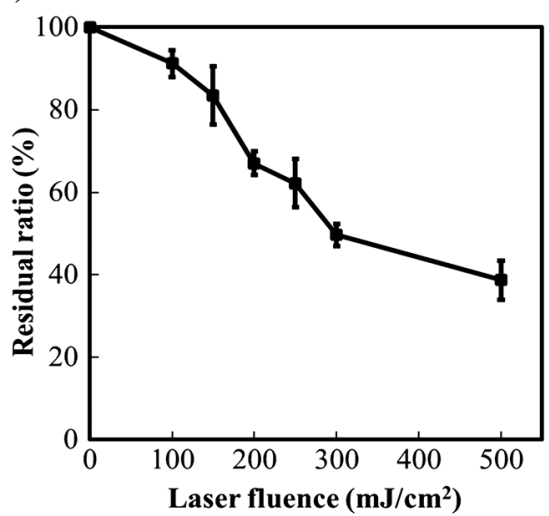

(b)

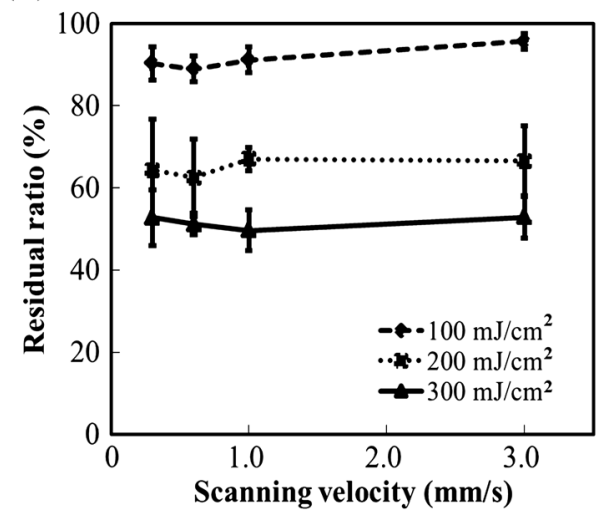

Fig. 3 Residual ratios of the microcapsules after laser irradiation. (a) Dependence of the residual ratio of microcapsules on the laser fluence. Laser scanning velocity was $1 \mathrm{~mm} / \mathrm{s}$. (b) Residual ratio with different laser scanning velocities under laser fluences of 100,200 , and $300 \mathrm{~mJ} / \mathrm{cm}^{2}$. Error bars indicate standard deviation $(n=5)$.

The microcapsules were spherical after laser irradiation; however, the roughness of the microcapsule shell was increased. The structure of the roughness may have been caused by partial melting followed by coagulation. The repetition rate of laser irradiation was $1 \mathrm{kHz}$; therefore, the steady increase of ambient temperature by heat accumulation is considered to be negligible. The melting might be caused by the transient increase in temperature by a laser pulse. The roughness was observed on the entire surface of the microcapsules, probably due to the multiple laser pulse irradiation in which the laser pulse overlap was 100 . The area of roughness increased 5 days after the irradiation, as shown in Fig. 5(b). The microcapsules that were not irradiated with the laser pulses are shown in Figs. 5(c) and 5(d), which were observed on the second and sixth days after the fabrication, respectively. In contrast to the results with the irradiated microcapsules, the microcapsules that were not irradiated had less rough surfaces even 6 days after fabrication.

Figure 6 shows the optical intensity distribution around a PLGA microcapsule, calculated using the 3-D FDTD method. Because the diameter of the laser spot in the above experiments was $300 \mu \mathrm{m}$, which was much larger than the average diameter of microcapsules, we have simulated the illumination of a plane

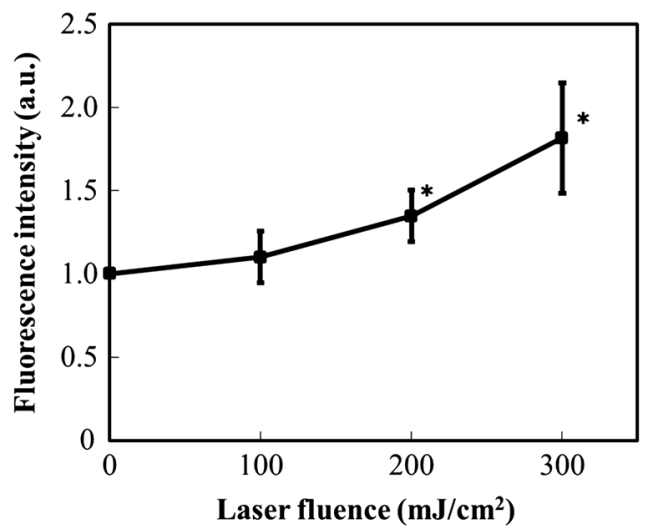

Fig. 4 Dependence of fluorescence intensity from released molecules on the laser fluence. The laser scanning velocity was $2 \mathrm{~mm} / \mathrm{s}$. Error bars indicate standard deviation $(n=5)$. * indicates that the fluorescence intensity is statistically significant to the value without laser irradiation $(p<0.01)$. wave. The diameter of the microcapsule is much larger than the incident wavelength $(800 \mathrm{~nm})$; this resulted in the lens focusing the laser energy, which has a focal spot distant from the shell surface. However, enhancement of the optical intensity in the shell was observed to increase beyond 10 in relation to the incident optical intensity.

\section{Discussion}

The experimental values of the microcapsule diameters were different from the theoretical values, specifically in the ultrasound
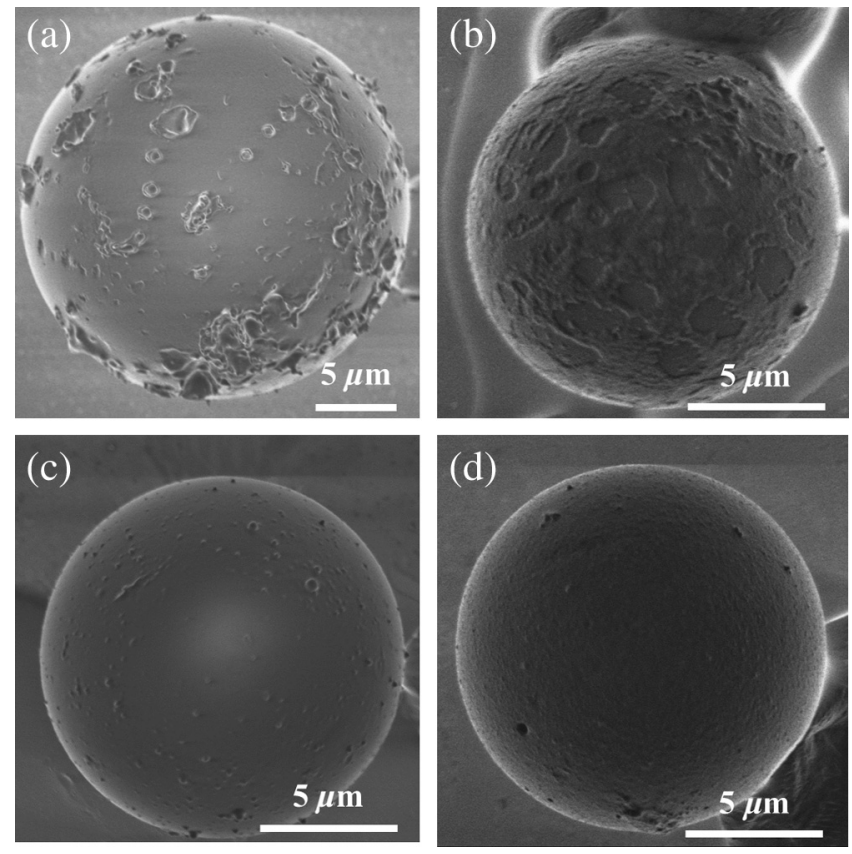

Fig. 5 SEM images of PLGA microcapsules. (a) Microcapsules 1 day after laser irradiation with femtosecond laser pulses at $200 \mathrm{~mJ} / \mathrm{cm}^{2}$ laser fluence and $1 \mathrm{~mm} / \mathrm{s}$ scanning velocity, corresponding to the pulse overlap of 100. (b) Microcapsules 5 days after laser irradiation with femtosecond laser pulses at $200 \mathrm{~mJ} / \mathrm{cm}^{2}$ laser fluence and $1 \mathrm{~mm} / \mathrm{s}$ scanning velocity. (c) Microcapsules without laser irradiation 2 days after fabrication. (d) Microcapsules without laser irradiation 6 days after fabrication. 


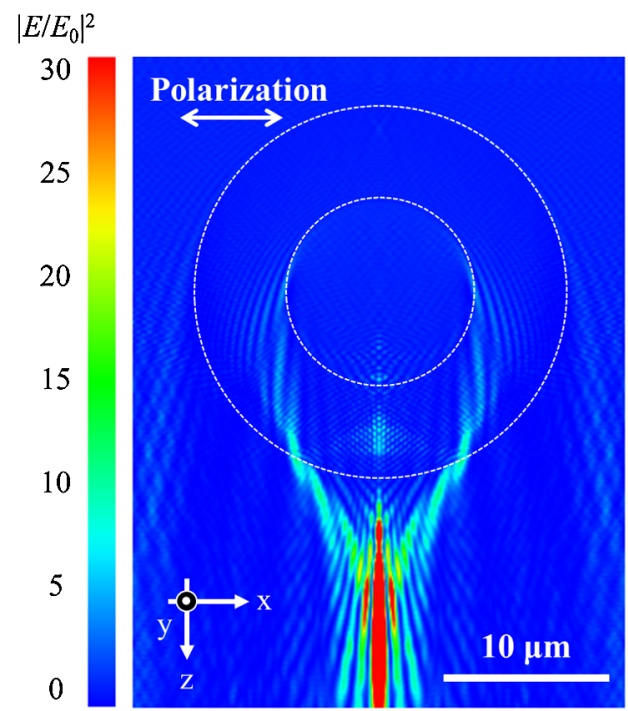

Fig. 6 Optical intensity distribution around a PLGA microcapsule on $x-z$ plane calculated by the FDTD method. The dashed circles indicate the internal and external diameters of the microcapsule. The microspheres were illuminated by a plane wave, with the wave vector in the $z$-direction. The incident wave is linearly polarized along the $x$-axis.

frequencies above $10 \mathrm{kHz}$ [Fig. 1(d)]. The velocity and viscosity of the solution in the nozzle are the key parameters for determining the diameter of the microcapsule. The flowing solution was split into droplets because of the nozzle vibration, which was controlled by an ultrasound transducer. The ease of splitting is dependent on the viscosity of the solution. It was found that the solution could not be split into droplets at higher nozzle vibration frequencies; this result can be explained by a similar case of a single nozzle system reported by Berkland et al. ${ }^{9}$

The residual ratio of the microcapsules after laser irradiation was dependent on the laser fluence [Fig. 3(a)], whereas the scanning velocity had a negligible effect on the residual ratio [Fig. 3(b)]. These results indicate that the rupture of the PLGA microcapsules was not influenced by the total dose of laser energy, but by the peak intensity of the laser pulse. Assuming the highest laser fluence in our study, $500 \mathrm{~mJ} / \mathrm{cm}^{2}$, the peak intensity of the pulse is estimated to be $6.25 \times 10^{12} \mathrm{~W} / \mathrm{cm}^{2}$, which is comparable with or lower than ablation thresholds for most of the transparent dielectric materials. ${ }^{20}$ The simulation result in Fig. 6 indicated that the enhancement of the optical intensity was obtainable in the shell of the microcapsule. The rupture of microcapsules was attributed to the nonlinear interaction of the enhanced peak intensity at the shell. It should be noted that the residual ratio in Fig. 3(a) did not show a nonlinear curve with the increase in laser fluence. This can be explained by the intensity distribution of the laser pulse. The spatial profile of the laser intensity in the experiment is presumed to be Gaussian, and it is expressed as follows:

$I(r)=I_{0} \exp \left(-\frac{2 r^{2}}{w_{0}^{2}}\right)$,

where $I_{0}, r$, and $w_{0}$ are the peak intensity, distance from the center of laser beam, and radius of the Gaussian beam, respectively. The beam radius $w_{1}$ at the intensity $I_{1}$ is written as $w_{1}=-\frac{w_{0}}{\sqrt{2}} \log \left(\frac{I_{1}}{I_{0}}\right)$.

Assuming the enhancement factor in the shell is 10 in relation to the incident intensity and the threshold for the rupture of the transparent materials is $10^{13} \mathrm{~W} / \mathrm{cm}^{2}$, the incident intensity that can reach the threshold after the enhancement is $10^{12} \mathrm{~W} / \mathrm{cm}^{2}$. Here, $I_{1}$ is $10^{12} \mathrm{~W} / \mathrm{cm}^{2}, w_{1}$ is estimated to be larger than $150 \mu \mathrm{m}$ when the laser intensity $I_{0}$ is greater than $4.1 \times 10^{12} \mathrm{~W} / \mathrm{cm}^{2}$, and the corresponding laser fluence is $329 \mathrm{~mJ} / \mathrm{cm}^{2}$. In this case, the laser intensity is higher than the threshold at all areas in the laser spot, suggesting that no dominant decrease in the residual ratio can be expected for laser fluences above this threshold. This estimation exhibits good agreement with the result shown in Fig. 3(a) and indicates that the optical intensity is a dominant factor for the rupture of microcapsules.

The release of the fluorescent molecules from the PLGA microcapsules is dependent on the rupture of microcapsules. Figure 7 shows the relationship between the adjusted/nonadjusted fluorescence intensities and the ruptured ratio (1residual ratio). The irradiation of laser pulses could cause photobleaching; therefore, photobleaching was measured separately and accounted for in the adjusted fluorescence intensity shown in the figure. The decrease in the fluorescence intensity caused by photobleaching was measured to be $6.2 \%, 10.1 \%$, and $15.8 \%$ with laser fluences 100,200 , and $300 \mathrm{~mJ} / \mathrm{cm}^{2}$, respectively. In Fig. 7, the fluorescence intensity shows a significant increase at $300 \mathrm{~mJ} / \mathrm{cm}^{2}$, which is a deviation from the extrapolation from the plots for lower fluences. This indicates the molecular release without a significant rupture of the microcapsule, which could not be observed using an optical microscope. Figure 8 shows an SEM image of a microcapsule irradiated by laser pulses at a laser fluence of $300 \mathrm{~mJ} / \mathrm{cm}^{2}$ and laser scanning velocity of $2 \mathrm{~mm} / \mathrm{s}$. The SEM image was obtained for microcapsules on the fifth day after laser irradiation. It should be noted that in microscale, laser ablation was observed on the shell of the microcapsule, which is difficult to identify using an optical microscope. The laser ablation on the shell was also observed in our previous study. ${ }^{19}$ If such

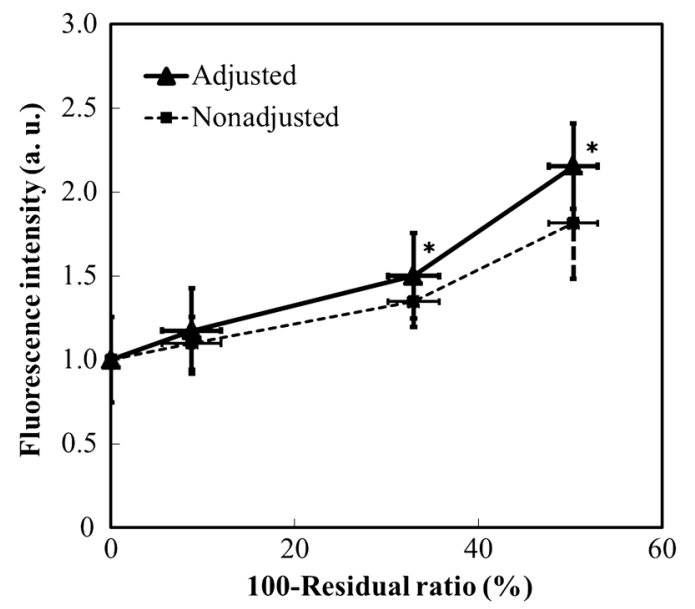

Fig. 7 Adjusted and nonadjusted fluorescence intensities versus that (1-residual ratio) derived from Figs. 3(a) and 4. Error bars indicate standard deviation $(n=5)$. * indicates that the adjusted fluorescence intensity is statistically significant to the value without laser irradiation $(p<0.01)$. 


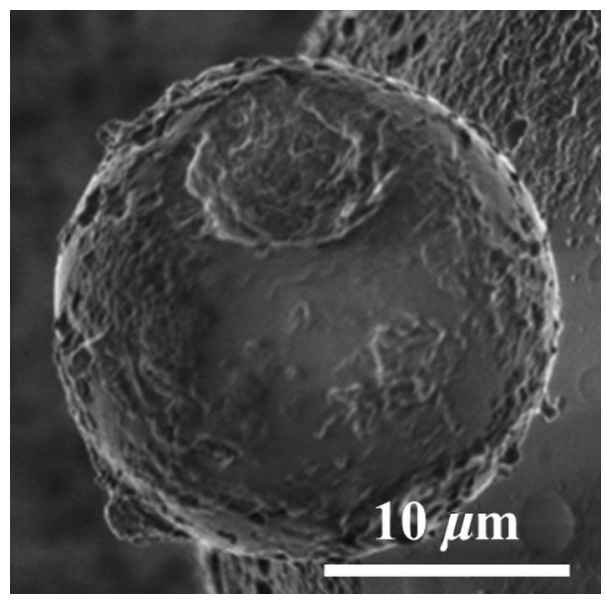

Fig. 8 SEM image of a PLGA microcapsule irradiated with laser pulses at laser fluence of $300 \mathrm{~mJ} / \mathrm{cm}^{2}$ and laser scanning velocity of $2 \mathrm{~mm} / \mathrm{s}$.

a microcrater penetrates as a through-hole to the internal core, the microcapsule can release encapsulated molecules without disappearing. At higher laser fluences, a strong enhanced optical field that is generated in a limited space might contribute to the release of the encapsulated molecules.

The effects of laser irradiation on the degradation rate of biodegradable polymers have been reported in many papers. Aguilar et al. ${ }^{21}$ reported the acceleration of PGA degradation after irradiation with nanosecond laser pulses (wavelength $193 \mathrm{~nm}$, pulse duration $35 \mathrm{~ns}$, and repetition rate $2 \mathrm{kHz}$ ). Hsu et al. ${ }^{22}$ discussed that the irradiation of nanosecond laser pulses (wavelength $248 \mathrm{~nm}$ and pulse duration $25 \mathrm{~ns}$ ) dissociated the $\mathrm{CO}-\mathrm{CO}_{2}$ bonds, resulting in the decrease in poly-Llactic acid crystallinity; $\mathrm{Hsu}$ and $\mathrm{Yao}^{23}$ also reported the acceleration of biodegradation. The irradiation of femtosecond laser pulses also induces a change in the degradability of the microcapsule, which is dependent on the wavelengths of the laser pulses. ${ }^{24}$ In Figs. 5 and 8, traces of partial melting were observed on the surface of the shell after laser irradiation, indicating that the polymer modification may affect the time profile of the release after laser irradiation. Although this study evaluated the transient burst release after laser irradiation, further investigation into the possibility of the sustained release after laser irradiation will facilitate realization of a more advanced laser-controlled drug release.

\section{Conclusion}

In this study, we demonstrated the release of encapsulated molecules from PLGA microcapsules triggered by femtosecond laser pulses. The PLGA microcapsules encapsulating fluorescence molecules were fabricated by a dual-coaxial nozzle system. The irradiation of femtosecond laser pulses enhanced the release of the molecules from the microcapsules, accompanied by a decrease in the residual ratio of the microcapsules. An SEM observation revealed the laser-irradiation-induced modification of the surface of the microcapsule shell, indicating the potential for sustained release as well as transient burst release.

\section{Acknowledgments}

This work was supported in part by KAKENHI Grant-in-Aid for Young Scientists (A) No. 26702019.

\section{References}

1. S. Mura, J. Nicolas, and P. Couvreur, "Stimuli-responsive nanocarriers for drug delivery," Nat. Mater. 12, 991-1003 (2013).

2. B. P. Timko, T. Dvir, and D. S. Kohane, "Remotely triggerable drug delivery systems," Adv. Mater. 22(44), 4925-4943 (2010).

3. A. P. Esser-Kahn et al., "Triggered release from polymer capsules," Macromolecules 44(14), 5539-5553 (2011).

4. C. Evora et al., "Relating the phagocytosis of microparticles by alveolar macrophages to surface chemistry: the effect of 1,2-dipalmitoylphosphatidylcholine," J. Controlled Release 51(2-3), 143-152 (1998).

5. U. Wattendorf et al., "Mannose-based molecular patterns on stealth microspheres for receptor-specific targeting of human antigen-presenting cells," Langmuir 24(20), 11790-11802 (2008).

6. R. Salem et al., "Treatment of unresectable hepatocellular carcinoma with use of Y-90 microspheres (therasphere): safety, tumor response, and survival," J. Vasc. Interventional Radiol. 16(12), 1627-1639 (2005).

7. Y. Xia and D. W. Pack, "Uniform biodegradable microparticle systems for controlled release," Chem. Eng. Sci. 125, 129-143 (2015).

8. C. Berkland et al., "Uniform double-walled polymer microspheres of controllable shell thickness," J. Controlled Release 96(1), 101-111 (2004).

9. C. Berkland, K. Kim, and D. W. Pack, "Fabrication of PLG microspheres with precisely controlled and monodisperse size distributions," J. Controlled Release 73(1), 59-74 (2001).

10. J. Panyam et al., "Polymer degradation and in vitro release of a model protein from poly (D,L-lactide-co-glycolide) nano- and microparticles," J. Controlled Release 92(1-2), 173-187 (2003).

11. S. Kamei et al., "New method for analysis of biodegradable polyesters by high-performance liquid chromatography after alkali hydrolysis," Biomaterials 13(13), 953-958 (1992).

12. H. Pistner et al., "Poly (l-lactide): a long-term degradation study in vivo. Part III: analytical characterization," Biomaterials 14(4), 291-298 (1993).

13. H. K. Makadia and S. J. Siegel, "Poly lactic-co-glycolic acid (PLGA) as biodegradable controlled drug delivery carrier," Polymers 3(3), 13771397 (2011).

14. M. K. Gupta et al., "3D printed programmable release capsules," Nano Lett. 15(8), 5321-5329 (2015).

15. J. Xi et al., "Au nanoparticle-coated, PLGA-based hybrid capsules for combined ultrasound imaging and HIFU therapy," J. Mater. Chem. B 3, 4213-4220 (2015).

16. Z. Deng et al., "Hollow chitosan-silica nanospheres as $\mathrm{pH}$-sensitive targeted delivery carriers in breast cancer therapy," Biomaterials 32(21), 4976-4986 (2011).

17. J. Ge et al., "Drug release from electric-field-responsive nanoparticles," ACS Nano 6(1), 227-233 (2012).

18. C. H. Chu et al., "Surface deformation of gold nanorod-loaded poly(dllactide-co-glycolide) nanoparticles after near infrared irradiation: an active and controllable drug release system," J. Mater. Chem. 20(16), 3260-3264 (2010).

19. M. Terakawa et al., "Near-infrared femtosecond laser-triggered nanoperforation of hollow microcapsules," Opt. Express 21(10), 1260412610 (2013).

20. C. B. Schaffer, A. Brodeur, and E. Mazur, "Laser-induced breakdown and damage in bulk transparent materials induced by tightly-focused femtosecond laser pulses," Meas. Sci. Technol. 12(11), 1784-1794 (2001).

21. C. A. Aguilar et al., "Direct micro-patterning of biodegradable polymers using ultraviolet and femtosecond lasers," Biomaterials 26(36), 76427649 (2005).

22. S. T. Hsu, H. Tan, and Y. L. Yao, "Effect of excimer laser irradiation on crystallinity and chemical bonding of biodegradable polymer," Polym. Degrad. Stab. 97(1), 88-97 (2012).

23. S. T. Hsu and Y. L. Yao, "Effect of drug loading and laser surface melting on drug release profile from biodegradable polymer," J. Appl. Polym. Sci. 130(6), 4147-4156 (2013).

24. A. Shibata, S. Yada, and M. Terakawa, "Biodegradability of poly(lacticco-glycolic acid) after femtosecond laser irradiation,” Sci. Rep. 6, 27884 (2016). 
Kazumasa Ariyasu received his BS and MS degrees from Keio University in 2014 and 2016, respectively. His research covers laser-based drug delivery and drug release.

Atsuhiro Ishii received his BS degree in electronics and electrical engineering from Keio University. He is a graduate school student at Keio University, Japan. His research interests include the mechanisms and applications of laser cell perforation.

Taiga Umemoto received his BS degree in electronics and electrical engineering from Keio University. $\mathrm{He}$ is a graduate school student at the Keio University, Japan. His research interests include laser-based drug release and the fabrication of tissue scaffolds.

Mitsuhiro Terakawa received his BS, MS, and $\mathrm{PhD}$ degrees from Keio University in 2003, 2005, and 2007, respectively. In 2007, he joined the Wellman Center for Photomedicine, Massachusetts General Hospital, as a research fellow. He joined Keio University in 2009. He is an associate professor at Keio University. His research interests include ultrafast laser processing, laser processing of biomaterials, laser drug delivery, and laser-based nanostructure formation. $\mathrm{He}$ is a member of SPIE. 